\section{Prevalencia de la infección por Helicobacter pylori en niños: estimando la edad de adquisición}

\author{
FRANCISCA JAIME ${ }^{1}$, ANDREA VILLAGRÁN ${ }^{2, a}$, \\ CAROLINA SERRANO ${ }^{2, b}$, JAIME CERDA ${ }^{3}$, PAUL R. HARRIS ${ }^{2}$
}

\section{Frequency of Helicobacter pylori infection in 144 school age Chilean children}

Background: A 73\% prevalence of Helicobacter pylori infection was estimated in adults in the 2003 Chilean National Health Survey. However, this infection is usually acquired during childhood. Aim: To determine the frequency of $H$. pylori infection in healthy Chilean children from a school in Santiago. Material and Methods: A cross sectional study in a privatel subsidized school in Santiago. Children aged less than 18 years were invited to participate. The parents of those who accepted answered a demographic survey and a stool sample was obtained from participants to detect $H$. pylori antigen using a monoclonal antibody ELISA kit. Results: We studied 144 students aged $10.6 \pm 3.1$ years (54\% females). Twenty six participants (18.1\%, 95\% CI: 12.4-24.9\%) had a positive test. Children from higher socioeconomic levels had a non-significant lower frequency of infection. No differences in the frequency of infection were observed by age, gender, household type or number of people living in it or history of breastfeeding. Conclusions: In this sample of children, an $18.1 \%$ frequency of $H$. pylori infection was observed.

(Rev Med Chile 2013; 141: 1249-1254).

Key words: Child; Helicobacter pylori: Prevalence.

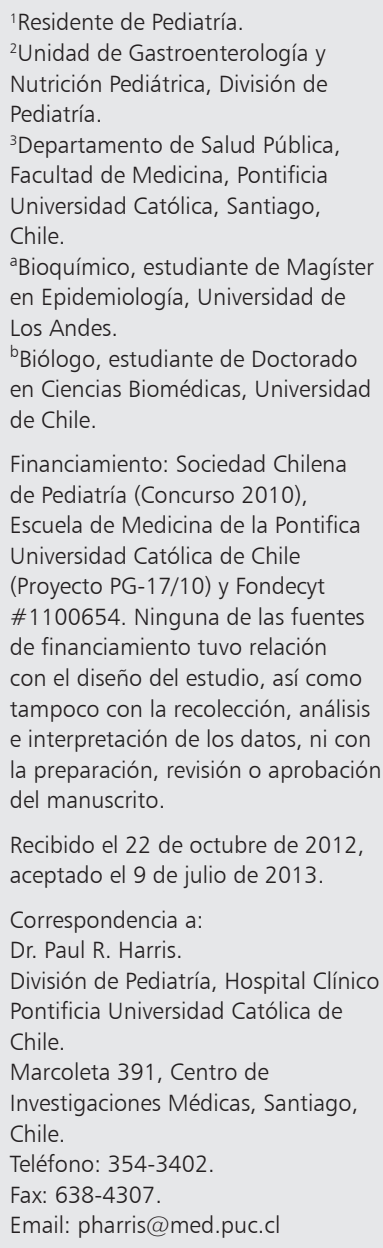

Financiamiento: Sociedad Chilena de Pediatría (Concurso 2010)

Escuela de Medicina de la Pontifica Universidad Católica de Chile (Proyecto PG-17/10) y Fondecyt \#1100654. Ninguna de las fuentes de financiamiento tuvo relación con el diseño del estudio, así como tampoco con la recolección, análisis e interpretación de los datos, ni con la preparación, revisión o aprobación del manuscrito.

Recibido el 22 de octubre de 2012, aceptado el 9 de julio de 2013.

Correspondencia a:

Dr. Paul R. Harris.

División de Pediatría, Hospital Clínico Pontificia Universidad Católica de

Chile.

Marcoleta 391, Centro de

Investigaciones Médicas, Santiago, Chile.

Teléfono: 354-3402.

Fax: 638-4307.

Email:pharris@med.puc.cl

L a bacteria Helicobacter pylori fue descrita por primera vez en 1875. Sin embargo, recién en 1982 fue redescubierta por Robin Warren y Barry Marshall ${ }^{1}$, iniciando tres décadas de profunda investigación en fisiopatología gástrica, con la apertura de campos no conocidos previamente como la inmunopatogénesis y la microbiología gástrica. Actualmente, la asociación entre $\mathrm{H}$. pylori con úlcera gástrica, duodenal, gastritis y cáncer gástrico ha sido fehacientemente establecida. Sin embargo, muchas áreas permanecen por ser dilucidadas, incluyendo el momento preciso de la adquisición de la infección.

La asociación entre H. pylori y cáncer gástrico es importante para nuestro país dado la alta prevalencia de cáncer gástrico en nuestro territorio ${ }^{2}$, que nos ubica entre los países con mayor mortalidad por esta causa a nivel mundial, junto con Costa Rica y Rusia ${ }^{3}$. Diferentes estudios han dado cuenta que entre 59 y $63 \%$ de la incidencia de cáncer gástrico se explica por $H$. pylori $i^{2,4}$, por lo que nuestro país representa un lugar prioritario para su estudio.

No obstante, es preocupante que si bien esta infección se manifiesta principalmente en el período de adultez, su contagio ocurre en la edad pediátrica ${ }^{5}$ hoy, a más de un cuarto de siglo de su redescubrimiento, sólo conocemos la prevalencia de infección por $\mathrm{H}$. pylori en adultos chilenos $(72,99 \%)^{6}$, pero no tenemos información sobre la prevalencia en niños, dato fundamental para la eventual toma de decisiones a nivel de salud pública en cuanto a estrategias de erradicación, vacunación u otras. 
Por tanto, esta investigación evaluó la prevalencia de la infección por $H$. pylori en escolares chilenos a través de la medición de antígenos en deposiciones, un test no invasivo de fácil aplicación y excelente rendimiento.

\section{Pacientes y Métodos}

\section{Población en estudio}

Se reclutaron niños sanos menores de 18 años de edad, matriculados en el Colegio Nueva Era Siglo XXI, ubicado en la comuna de La Florida, en la zona urbana de Santiago. Este colegio, de financiamiento particular/subvencionado, tuvo una matrícula el año 2011 de 1.586 alumnos, hombres y mujeres, con 41 cursos desde kínder a IV medio, con 3 a 4 cursos por nivel, cada uno con entre 36 a 44 alumnos. Los alumnos cursan jornada escolar completa, por lo que se alimentan en el colegio, ya sea comida preparada en el mismo, o traída desde sus hogares. Además cuenta con servicios sanitarios básicos.

Este proyecto fue aprobado por el Comité de Ética de la Facultad de Medicina de la Pontificia Universidad Católica de Chile y cumple con la Declaración de Helsinki.

\section{Criterios de inclusión y exclusión}

Se consideró como criterios de inclusión: tener nacionalidad chilena y ser hijo de padres chilenos, dado que se conoce que niños inmigrantes podrían presentar prevalencias de infección más parecidas a las de su país de origen ${ }^{19}$; edad menor o igual a 18 años; estar matriculado en el colegio en estudio; firma de consentimiento informado por parte de los padres y de asentimiento por parte del niño para participar en el estudio. Fueron criterios de exclusión: ingesta en el último mes de antibióticos, inhibidores de la bomba de protones, inhibidores de receptor histaminérgico o bismuto, dado que existe literatura que muestra una disminución del rendimiento del test en estas condiciones ${ }^{11,12}$.

\section{Encuesta}

Posterior a reuniones de trabajo e información a las autoridades y profesores del colegio, a los padres/apoderados interesados en participar se les entregó la encuesta y el consentimiento informado/asentimiento del menor para ser respondidos en su hogar, siendo devuelto ambos documentos posteriormente a los investigadores, resguardando la confidencialidad de los datos. La encuesta tuvo una primera parte de datos demográficos del menor (nombre, fecha de nacimiento, curso, domicilio); una segunda parte con preguntas cerradas: tipo de vivienda (casa vs departamento), ¿cuántas personas de 18 o más años viven en el hogar?, ¿cuántas personas menores de 18 años, incluyendo al niño(a), viven en la casa?, ¿el niño(a) comparte la pieza? (sí/no), ¿el niño(a) comparte la cama? (sí/no), ¿el niño(a) recibió lactancia materna? (sí/no). Si su respuesta es sí: ¿por cuántos meses? En la tercera parte de la encuesta se preguntó por antecedentes educacionales de los padres y trabajo actual, para determinar el nivel socioeconómico. Esto último se realizó a través de la metodología ESOMAR, la que ha sido utilizada previamente en estudios epidemiológicos como la Encuesta Nacional de Salud $2003^{15}$. A partir de los datos entregados se determinaron los niveles: A (muy alto), B (alto), CA (medio-alto), CB (medio), D (medio-bajo) y E (bajo), este último equivalente al nivel de pobreza según la Encuesta Casen ${ }^{16}$.

\section{Evaluación de la presencia/ausencia de infección por H. pylori}

La infección por $H$. pylori fue evaluada a través de la determinación monoclonal de antígenos de la bacteria en una muestra de deposiciones (Premier Platinum HpSA, Meridian Diagnostics, Ohio, EEUU). Este test diagnóstico presenta muy buena sensibilidad y especificidad (94 y 97\%, respectivamente), según datos publicados por nuestro grupo ${ }^{20}$ y otros.

Para la obtención de la muestra, se les entregó a los padres un kit que incluyó: un receptáculo para deposiciones, un frasco contenedor, guantes desechables y espátula con un instructivo para la toma. Se les indicó a los padres que debían guardar la muestra en el refrigerador de su hogar hasta ser entregada en el colegio, sin pasar más de 24 $\mathrm{h}$, donde se mantuvo refrigerado, siendo retirado diariamente y congelado en nuestro laboratorio a $-80^{\circ} \mathrm{C}$ hasta su análisis.

El análisis se realizó según las instrucciones del fabricante. Para la determinación de cada muestra, $500 \mu \mathrm{L}$ de solución tampón fosfato 10 $\mathrm{mM}$ con timerosal $0,02 \%$, y una muestra de 5-6 $\mathrm{mm}$ de diámetro de deposiciones fueron mezcladas mediante uso de vortex durante 15 segundos. Posteriormente, se añadió $100 \mu \mathrm{L}$ de la muestra diluida al micropocillo con anticuerpos monoclo- 
nales específicos para $H$. pylori. Se añadió 1 gota de conjugado enzimático (anticuerpos monoclonales específicos para $H$. pylori conjugados a peroxidasa de rábano en una solución tampón Tris $50 \mathrm{mM}$, conteniendo timerosal 0,02\%). Se agitó y mezcló la placa con movimientos giratorios durante 30 segundos. Se incubó la placa sellada durante una hora a temperatura ambiente. Luego se realizó lavado por 5 veces con tampón fosfato $180 \mathrm{mM}$, con timerosal $0,02 \%$. Se añadió a los micropocillos 2 gotas (aproximadamente $100 \mu \mathrm{L}$ ) de tampón citrato-acetato, con peróxido de urea y 3,3',5,5' etrametilbenzidina. Se agitó durante 30 segundos y se incubó a temperatura ambiente por $10 \mathrm{~min}$. Finalmente, se agregó dos gotas de la solución de ácido fosfórico $1 \mathrm{M}$, se agitó la placa por 30 segundos y se leyó con espectrofotómetro a $450 \mathrm{~nm}$. Los resultados se interpretaron según los rangos aportados por el fabricante.

\section{Análisis estadístico}

Los resultados fueron analizados con el programa estadístico SPSS 17.0 (IBM(C Company). Para la asociación de la presencia de $H$. pylori con variables categóricas se usó test de $\chi^{2}$ o Fisher según correspondiera, y para variables numéricas, T-Student para muestras independientes. Además, para evaluar variables confundentes se realizó regresión logística binaria. Se consideró diferencia significativa un $\mathrm{p}<0,05$.

\section{Resultados}

\section{Características demográficas}

Se reclutaron 144 alumnos desde kínder a IVo medio entre marzo y noviembre de 2011. La edad promedio de los participantes fue 10,6 $\pm 3,1$ años.
Setenta y siete participantes eran mujeres (54,2\%). La distribución por edad se describe en la Figura 1.

\section{Presencia de H. pylori}

Se recibió muestras de la totalidad de los alumnos reclutados. El test de antígeno en deposiciones resultó positivo en 26 niños (prevalencia 18,1\%; IC 95\%: 12,41-24,98\%). Dos alumnos entregaron la muestra sin identificación, por lo que sólo se analizó en esos casos la presencia/ausencia de infección.

\section{Encuesta}

De la totalidad de los participantes, 126 $(87,5 \%)$ alumnos entregaron además la encuesta. La tasa de respuestas para cada una de las preguntas varió desde 89,6 a $100 \%$ y sus resultados se muestran en la Tabla 1. La distribución por niveles socioeconómicos se muestra en la Figura 2. Destacó que $86,3 \%$ de los participantes perteneció al nivel socioeconómico medio (grupos medio alto, medio y medio bajo). No hubo participantes en rango de pobreza (Nivel E).

\section{Relación presencia/ausencia de H. pylori y encuesta}

Se encontró menor prevalencia de la infección en los niveles socioeconómicos más altos (Tabla 2), lo que no alcanzó significancia estadística. Dado que 18 participantes no entregaron la encuesta, no se dispone de los datos de NSE para ellos, por lo que se estableció 4 posibles escenarios.

Escenario 1: Los participantes que no contestaron la encuesta pertenecerían al NSE alto. En este escenario, se mantiene la tendencia a menor prevalencia en niveles más altos, sin alcanzar significancia estadística $(p=0,768)$. Escenario 2: Todos los participantes que no contestaron la encuesta

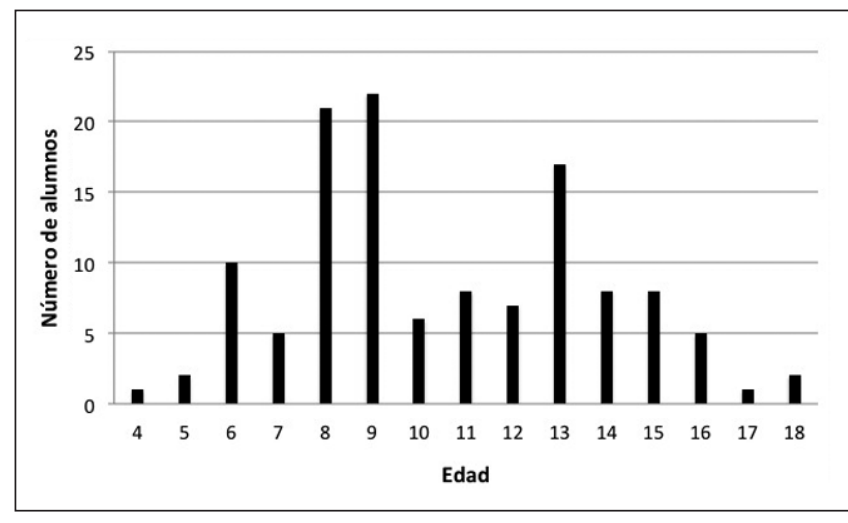

Figura 1. Distribución de participantes según edad. $P=0,022$, Test Kolmogorov-Smirnoff. 
Tabla 1. Características de la población estudiada

\begin{tabular}{|lccc|}
\hline Característica & H. pylori (-) & H. pylori (+) & valor p \\
\hline Edad en años, promedio (DE) & $10,6(3,1)$ & $10,3(3,3)$ & 0,669 \\
\hline Mujeres, n (\%) & $67(57,8)$ & $10(38,4)$ & 0,085 \\
\hline Vive en casa vs departamento, n (\%) & $97(95)$ & $23(95,8)$ & 1 \\
\hline No de personas < 18 años, promedio (DE) & $2,1(1,0)$ & $1,9(0,7)$ & 0,518 \\
\hline No de personas > 18 años, promedio (DE) & $2,7(1,1)$ & $3(1,4)$ & 0,282 \\
\hline Años de estudio de la madre, promedio (DE) & $14,7(2,4)$ & $14,1(3,6)$ & 0,361 \\
\hline Recibió leche materna, n (\%) & $98(96,1)$ & $21(91,3)$ & 0,305 \\
\hline Por cuánto tiempo, promedio (DE) & $9,3(7,1)$ & $12(7,7)$ & 0,123 \\
\hline Comparte cama, n (\%) & $10(9,8)$ & $2(9,1)$ & 0,591 \\
\hline Comparte pieza, n (\%) & $36(35,3)$ & $9(37,5)$ & 0,508 \\
\hline
\end{tabular}

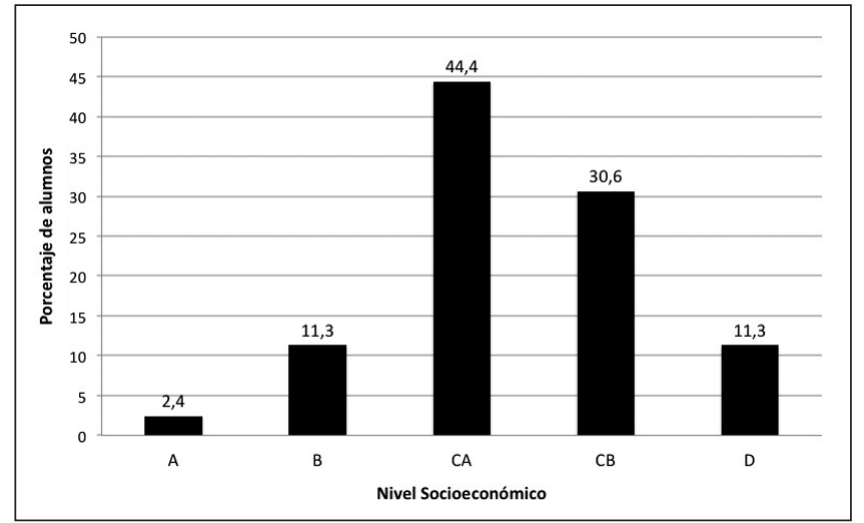

Figura 2. Distribución de los participantes según nivel socioeconómico. $A=$ Muy alto; $B=$ Alto; $\mathrm{CA}=$ Medio alto; $\mathrm{CB}=$ Medio; $\mathrm{D}=$ Medio bajo.
Tabla 2. Positividad de las muestras por nivel socioeconómico (NSE)

\begin{tabular}{|ccc|}
\hline NSE & \% positividad & IC $\mathbf{9 5 \%}$ \\
\hline$A+B+C A$ & 16,7 & $8,1-23,32$ \\
\hline$C B$ & 21,1 & $8,1-34,1$ \\
$D$ & 21,4 & $0-42,9$ \\
\hline
\end{tabular}

Valor $p=$ No significativo entre los grupos.

pertenecerían al NSE bajo. En este caso, persiste la mayor prevalencia en el NSE medio, sin alcanzar significancia estadística $(\mathrm{p}=0,85)$. Escenario 3: Todos los participantes que no contestaron la encuesta y que son $H$. pylori $(+)$ pertenecerían al NSE bajo, aquí se mantiene la tendencia, sin alcanzar significancia estadística $(\mathrm{p}=0,09)$. Finalmente, escenario 4 , todos los participantes que no contestaron la encuesta y son $H$. pylori $(+)$ pertenecerían al NSE alto, en cuyo caso habría menor prevalencia en el NSE bajo, sin alcanzar significancia estadística $(\mathrm{p}=0,38)$.

No se encontró diferencia en la positividad de las muestras según edad ni por nivel escolar del niño ni de los padres, tipo de habitación, número de personas que habitan en la misma residencia con el alumno ni antecedente de lactancia materna.

\section{Discusión}

En este estudio se encontró una prevalencia de infección por $H$. pylori de $18,1 \%$ a través de la determinación de antígenos en deposiciones. A nuestro conocimiento, este es el primer trabajo que evalúa la prevalencia de la infección en escolares sanos en nuestro país, siendo los estudios previos, realizados en población enferma que consulta por patología digestiva. 
Se sabe que la presencia de $H$. pylori en un individuo tiene relación con el nivel socioeconómico de éste, y a nivel poblacional, se asocia además con el nivel de desarrollo del país de residencia. Así, países como Gambia que se ubican en nivel de desarrollo bajo ${ }^{7}$, tienen una prevalencia de infección por $H$. pylori en niños que llega a $84 \%{ }^{8}$, mientras que países con desarrollo muy alto como Japón, presentan una prevalencia inferior a 10\% ${ }^{9}$. Chile se ubica entre los países con desarrollo alto según la clasificación de la $\mathrm{ONU}^{7}$, junto a otros como Brasil y Turquía, que presentan una prevalencia reportada en niños de $47,5 \%{ }^{10}$ y $23,9 \%{ }^{4}$, respectivamente.

En la Encuesta Nacional de Salud del año $2003^{15}$, la prevalencia de infección por $H$. pylori en adultos fue de $73 \%$. Sin embargo, en el análisis por subgrupos del mismo estudio, publicado por Ferreccio y cols. ${ }^{6}$, la prevalencia de infección en el grupo etario de 17 a 24 años, fue de 62,0\% (IC 95\%: 53,5-69,9\%). Este mismo estudio determinó además que la prevalencia a nivel local de infección por $H$. pylori se afecta por la tasa de mortalidad local por cáncer gástrico, con una prevalencia de infección ajustada por este dato, para este mismo grupo etario en la ciudad de Santiago, de 49,3\% (IC 95\%: 36,7-62,0\%). A partir de lo anterior, los resultados de nuestro estudio se acercarían a los reportados por Ferreccio y cols. La Encuesta Nacional de Salud del año 2009-2010²4 no determinó prevalencia de infección por $H$. pylori.

El estudio de prevalencia del 2003 se realizó a través de serología para $H$. pylori (IgG), que en ese caso reportó una buena sensibilidad $(87,5 \%)$, pero baja especificidad (62\%) al compararlo con histología como gold standard. En ese sentido, la menor prevalencia encontrada en nuestro estudio podría explicarse porque nuestro trabajo se realizó con un test que presenta alta sensibilidad y especificidad, con menor cantidad de falsos positivos. Otra explicación hipotética es que las generaciones de chilenos que actualmente se encuentran en la etapa escolar presentan menor prevalencia de infección que sus antecesores dado las mejores condiciones de higiene y desarrollo del país, lo que es consistente con lo reportado en otros países en relación a mejoría en higiene y disminución de prevalencia en generaciones sucesivas ${ }^{5}$.

En nuestra opinión, el colegio seleccionado es representativo de los colegios de clase media de Santiago, con financiamiento particular/subven- cionado, que representa $58,2 \%$ de la matrícula de la Región Metropolitana ${ }^{25}$, con niños provenientes a su vez de diferentes niveles socioeconómicos, pero principalmente NSE medio. Adicionalmente, encontramos una tendencia hacia una menor prevalencia en los niveles socioeconómicos más altos, lo que concuerda con lo encontrado en estudios previos ${ }^{7-9}$, aunque en nuestro estudio no alcanzó significancia estadística. Los análisis de los distintos posibles escenarios, considerando los pacientes que no reportaron NSE, no cambiaron significativamente estas conclusiones, lo que podría explicarse por el bajo poder de la muestra para esta comparación.

Es importante continuar el estudio de la epidemiología de la infección por $H$. pylori particularmente en nuestro país, ya que la implementación de medidas de salud pública, tanto para prevenir la infección por $H$. pylori (vacunación), como para decidir el momento óptimo de un eventual tratamiento (erradicación) a nivel de población sana, podría traducirse a largo plazo en una disminución de la prevalencia de cáncer gástrico en nuestro país. Esto necesariamente deberá considerar la prevalencia de la infección en la niñez, para evitar vacunar una población ya expuesta a la bacteria y para evitar erradicar $H$. pylori de una población aún no infectada. Pero al desconocer la prevalencia en edades pediátricas y los factores asociados a ésta, es difícil estimar a qué grupo de menores deben enfocarse dichas medidas. Adicionalmente, en cuanto a la erradicación, el conocimiento tanto de la prevalencia por edades así como la tasa de eliminación de la infección permitiría el desarrollo de campañas que enfoquen el uso de recursos en sujetos ya infectados.

Nuestro estudio presentó algunas limitaciones, principalmente en el muestreo, que no fue aleatorio, sino por conveniencia a partir de la disposición de los padres a participar. Además, al pertenecer esta población mayoritariamente al nivel socioeconómico medio, estos resultados no necesariamente son representativos de escolares pertenecientes a otros NSE.

En conclusión, en este estudio encontramos una prevalencia de infección por $H$. pylori en escolares sanos, de $18,1 \%$, similar a la de países con el mismo nivel de desarrollo socioeconómico, lo que sugiere que, considerando los datos existentes de infección en población adulta, la mayor parte de la infección por $H$. pylori se adquiere en la segunda década de la vida. 


\section{Referencias}

1. Center for Disease Control. Helicobacter pylori and ulcer peptic disease. Disponible en http://www.cdc.gov/ulcer/ history.htm [Consultado el 20 de julio de 2012].

2. Ministerio de Salud. Guía Clínica de Cáncer Gástrico. Santiago. Minsal, 2006.

3. American Cancer Society. Global Cancer Facts \& Figures. Disponible en http://www.americancancersociety.com. [Consultado el 20 de julio de 2012].

4. Rollán A, Ferreccio C, Gederlini A, Serrano C, Torres J, Harris P. Non-invasive diagnosis of gastric mucosal atrophy in an asymptomatic population with high prevalence of gastric cancer. Word Journal of Gastroenterology 2006; 44 (12): 7172-8.

5. Azevedo N, Huntington J, Goodman K. The Epidemiology of Helicobacter pylori and Public Health Implications. Helicobacter 2009; 14 (Suppl 1): 1-7.

6. Ferreccio C, Rollán A, Harris P, Serrano C, Gederlini A, Margozzini P, et al. Gastric Cancer is Related to Early Helicobacter pylori infection in a high prevalence country. Cancer Epidemiol Biomarkers Prev 2007; 16 (4): 662-7.

7. Organización de Naciones Unidas. Human Development Report 2009. Disponible en http://hdr.undp.org/en/ reports/global/hdr2009/ [Consultado el 20 de julio de 2012].

8. Costa A, Figueiredo C, Touati E. Pathogenesis of Helicobacter pylori infection. Helicobacter 2009; 14 (Suppl 1): 15-20.

9. Naito Y, Shimizu T, Haruna H, Fujii T, Kudo T, Shoji $\mathrm{H}$, et al. Changes in the presence of urine Helicobacter pylori antibody in Japanese children in three different age groups. Pediatr Int 2008; 50: 291-4.

10. Rodrigues M, Queiroz D, Rodrigues R, Rocha A, Luz C, Braga L. Prevalence of Helicobacter pylori infection in Fortaleza, Northeastern Brazil. Rev Saúde Pública 2005; 39 (5): 247-9.

11. Gisbert JP, de la Morena F, Abraira V. Accuracy of monoclonal stool antigen test for the diagnosis of $H$. pylori infection: a systematic review and meta-analysis. Am J Gastroenterol 2006; 101 (8): 1921-30.

12. Broussard Ch, Goodman K, Phillips C, Smith M, Fischbach, Day S, Aragaki C. Antibiotics taken for other illnesses and spontaneous clearance of Helicobacter pylori infection in children. Pharmacoepidemiology and drug safety 2009; 18: 722-9.

13. Sykora J, Siala K, Varvarovská J, Pazdiora P, Pomahacová R, Huml M. Epidemiology of Helicobacter pylori infection in Asymptomatic Children: A prospective
Population-Based Study from Czech Republic. Application of a Monoclonal-Based Antingen-in-Stool Enzyme Immunoassay. Helicobacter 2009; 14: 286-97.

14. Silva I, Mendoza R, Costa G, Costa GT, Valle N, de Oliveira S, Boccio J, et al. Validity of an epidemiologic instrument for H. pylori among dyspeptic patients. Rev Saúde Pública 2009; 43: 1-7.

15. MINSAL. Resultados I Encuesta Nacional de Salud. Disponible en http://epi.minsal.cl/epi/html/invest/ENS/ InformeFinalENS.pdf [Consultado el 20 de julio de 2012].

16. Adimark. El Nivel Socioeconómico Esomar. Manual de Aplicación. [Online] 2006. http://www.adimark.cl. [Consultado el 20 de julio de 2012].

17. Monteiro L, Oleastro M, Lehours P, Mégraud F. Diagnosis of Helicobacter pylori infection. Helicobacter 2009; 14 (Suppl 1): 8-14.

18. Malfertheiner P, Megraud F, O’Morain C, Atherton J, Axon A, Bazzoli F, et al. Management of Helicobacter pylori infection: the Maastricht IV/ Florence Consensus Report. Gut 2012; 61: 646-64.

19. Cherian S, Burgner D, Carson C, Sanfilippo F, Cook A, Forbes D. Diagnosis of Helicobacter pylori infection in a High-prevalence Pediatric Population: A comparison of 2 Fecal Antigen Testing Methods and Serology. Journal of Pediatric Gastroenterology and Nutrition 2008; 47: 130-5.

20. González CG, Serrano C, Harris PR. Diagnóstico de la infección por Helicobacter pylori en niños mediante la detección de antígenos en deposiciones. Rev Med Chile 2007; 135: 182-8.

21. Okuda M, Miyashiro E, Booka M, Tsuji T, Nakazawa T. Helicobacter pylori colonization in the first 3 years of life in Japanese Children. Helicobacter 2007; 12: 324-7.

22. Kori M, Goldstein E, Granot E. Helicobacter pylori infection in young children detected by a monoclonal stool antigen immunoassay. The Pediatric Infectious Disease Journal 2009; 28 (2): 157-8.

23. Gisbert JP, Abraiva V. Accuracy of Helicobacter pylori diagnostic tests in patients with bleeding peptic ulcer: a systematic review and meta-analysis. Am J Gastroenterol 2006; 101 (4): 848.

24. Minsal. Encuesta Nacional de Salud ENS Chile 20092010. Disponible en http://www.minsal.gob.cl/portal/ docs/page/minsalcl/g_home/submenu_portada_2011/ ens2010.pdf [Consultado el 20 de julio de 2012].

25. Mineduc. Estadísticas de la Educación 2011. Disponible en http://centroestudios.mineduc.cl/index. php?t=96\&i=2\&cc=2044\&tm $=2$ [Consultado el 31 de marzo de 2013]. 